\title{
EARLIEST ARRIVAL EVACUATION PLANNING: TWO-TERMINAL CASE
}

\author{
Ram Uday Sah ${ }^{1}$, Shree Ram Khadka ${ }^{2}$ \\ ${ }^{1}$ Ratna Rajya Laxmi Campus (TU) \\ Email Address: ruday760@gmail.com \\ ${ }^{2}$ Central Department of Mathematics (TU) \\ Email Address: $\underline{\text { shreeramkhadka@gmail.com }}$
}

\begin{abstract}
Evacuation planning problem can be considered as a dynamic flow problem on the dynamic network. In a dangerous situation, as many individuals as possible should be rescued from a dangerous zone to a safety zone as quickly and efficiently as possible. The earliest arrival flow problem is to send a maximal amount of dynamic flow reaching the safety zone sink not only for the given time horizon, but also for any earlier moment of the time horizon. In this paper we discuss the optimization formulation of the earliest arrival evacuation planning problem with efficient solution procedure.
\end{abstract}

Keywords: Evacuation problem, Flow over time, Earliest Arrival flow.

\section{Introduction}

People are suffering from various types of natural and human made disasters e.g. earthquake, landslides, industrial accidents etc. So we need a very good planning for the disaster management that initiates all aspects of planning responding to disasters, including both pre and post-disaster activities which are in short Preparedness, Planning, Response and Recovery (PPRR). In a case of emergency, we need to evacuate some huge building, a stadium or a region. There is a maximum number of people that can enter the particular entity per time unit together with a corresponding transit time. The objective is to evacuate the maximum number of evacuees at each time unit out of the building or region which we call a source to the safety zone which is called a sink.

Evacuation planning based on static network flow which do not consider a significant transit time has been studied in the last century. A network consists of nodes arcs. Arcs connect the nodes. Each node models a town, a factory, a computer, a disaster zone etc. and the arc corresponds to the connection between them. Examples of arcs are a street, a cable etc. To each arc a capacity is assigned, which bounds the amount that can be transported on this arc. In a dynamic network, each arc has a transit time, which models the time it takes to traverse the arc. Dynamic flows also called flows over time. The capacity of an arc then defines the amount that may enter the arc at each discrete time step.

In this paper, we discuss the earliest arrival of the evacuation planning problem based on the dynamic network flow with optimization formulation with efficient solution procedure. The paper is organized as follows: we discuss a very brief literature of the problem in Section 2. We give the optimization formulation of the maximum dynamic flow evacuation planning problem in Section 3, Time expanded graph in Section 4, earliest arrival flow problem in Section 5 and complexity in Section 6. The final section concludes the paper.

\section{Literature Review}

The maximum dynamic flow problem (MDFP) was first studied by Ford and Fulkerson[8] with the goal of sending the maximum amount of flow (evacuees) from source $s$ i.e. a disastrous area to a sink $\mathrm{z}$ i.e. a safe area in a given time horizon T. They solved the problem optimally by presenting the 
temporally repeated flow technique. They also introduced the time expanded network and demonstrated that the general MDFP is equivalent to the static flow in the time expanded network. Gale [10] introduced the earliest arrival flow problem which is maximal in every time period t, $0 \leq$ $t \leq T$.

Minieka [18] and Wilkison [16] were the first to discuss the problem of earliest arrival flows which sends the evacuees to the sink as early as possible and designed algorithms for finding earliest arrival flows. Both algorithms relay on the successive shortest paths from $\mathrm{s}$ to $\mathrm{z}$ in the residual network. Hope and Tardos[11] presented polynomial time approximation algorithm for earliest arrival flow problem. As shown by Zadeh there are families of graphs that require the successive shortest path algorithms to have a Pseudo - polynomial number of iterations. The network given by Zadeh can be interpreted as instances of the Earliest Arrival Flow problem (EAFP), requiring the algorithm to need $\Omega(\mathrm{T})$ iterations and it is still an open problem whether the earliest arrival flow problem can be solved in polynomial time. In 2002, Fleischer and Skutella gave another fully polynomial time approximation scheme for earliest arrival flows. They allow the flow that should arrive at the sink by time t, to be an earliest arrival flow, to arrive at the sink with certain lateness. They developed condensed timeexpanded networks to get this approximation. Baumann and Skutella[2]considered a modified earliest arrival flow problem. They examined networks with several sources and sinks, where to each source and each sink a certain supply and demand is assigned. A feasible dynamic flow on this problem must satisfy these supplies and demands as additional constraints.

\section{Formulation of Maximal Dynamic Flow Problem}

Given a directed graph $\mathrm{G}=[\mathrm{N}, \mathrm{A}]$, Where $\mathrm{N}$ and $\mathrm{A}$ have their usual meaning. To each arc $(\mathrm{x}, \mathrm{y}) \epsilon \mathrm{A}$, we associates it with non negative integers denoted by $(\mathrm{x}, \mathrm{y})$ and $\alpha(\mathrm{x}, \mathrm{y})$ called the capacity and transit times of the $\operatorname{arcs}(\mathrm{x}, \mathrm{y}) \in A$. Let $0,1,2, \ldots, \mathrm{T}-1, \mathrm{~T}$ be the given discrete time period. Let $f(x, y, t)$ denotes the given discrete time period. Let $f(x, y, t)$ denotes the amount of flow that leaves $\mathrm{x}$ along $(x, y)$ at, consequently it reaches to the node $y$ at the time $t+\alpha(x+y)$. Also we have $f(x, y, t)$ is the hold over at $x$ from $y$ to at timet $=\alpha(x, y)$. Also we have $f(x, y, t)$ is the hold over at $x$ from $\mathrm{t}$ to $\mathrm{t}+1$.If $v(T)$ is net flow leaving source $\mathrm{s}$ and entering sink $\mathrm{z}$ during time periods 0 to1,1 to $2, \ldots$. T-1 to T. Then The L.P. formulation of maximal dynamic flow problem described by Ford and Fulkerson [8] is

Maximize $v(\mathrm{~T})$

$\sum_{t=0}^{T} \sum_{y \in N}[f(x, y, t)-f(y, x, t)-\alpha(y, x)]=v(T)$, for $x=s$

$\sum_{t=0}^{T} \sum_{y \in N}[f(x, y, t)-f(y, x, t-\alpha(y, x)]=v(T)$, for $x \neq s, z ; \mathrm{t}=0,1,2, \ldots \ldots ., \mathrm{T}$

$\sum_{t=0}^{T} \sum_{y \in N}[f(x, y, t)-f(y, x, t-\alpha(y, x)]=-v(T)$, for $x=z$

$0 \leq f(x, y, t) \leq c(x, y)$ For all $t \in\{0,1,2, . \mathrm{T}\}$ and $(x, y) \in A$ 


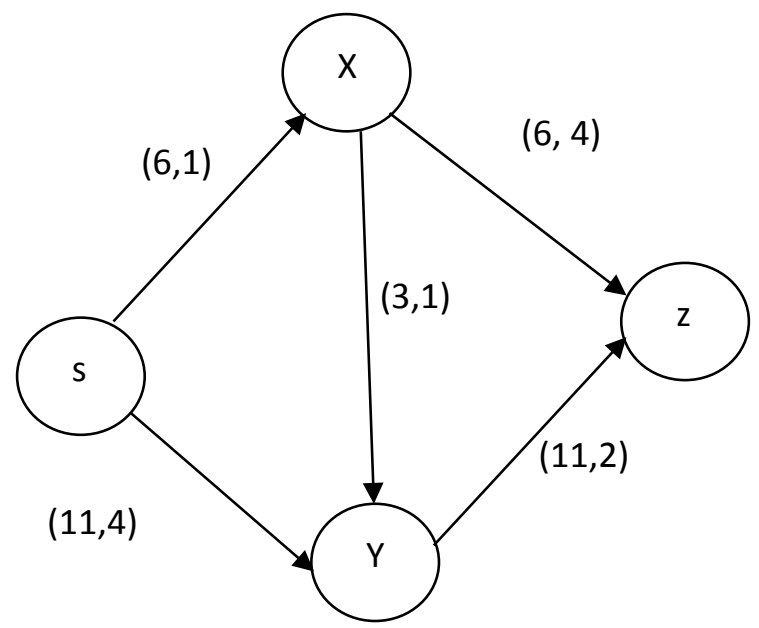

Fig 1 Dynamic Network Flow

\section{Time-Expanded Graph}

We have considered parameter capacity of the arcs and the travel time on that arc in the discussion of the network flow. Flows depending over time are more complex than the static flows, because the flow is specified for all times. There is no polynomial time algorithm to solve the min - cost flow problem over time with given supply. The problem can be easily solved by static flows if we search a flow that sends the total supply over time into the sink within a given time horizon $T$. If all transit times are integral, Algorithm for static flow problem in an extended network can be apply to this problem. This is called time - expanded network in which the flow over time problem can be solved in pseudo-polynomial time using static flow algorithm.

Ford and Fulkerson (1958)[8]introduced time-expanded network which is a static network obtained by an expansion of the dynamic network. A dynamic flow through the dynamic network $G=$ $(N, A, T)$ corresponds to a static flow in the time - expanded network $\mathrm{D}(\mathrm{T})$ where $\mathrm{N}$ is the set of nodes and $\mathrm{A} \subset N X N$ is the set of arcs which have non-negative flow capacities and non-negative integral travel times as their attributes. Time-expanded network are powerful tool to solve dynamic network flow problems, they allow applying the solution methods for static network flow problems to dynamic network flow problems.

The time expanded graph of the dynamic network with Time-Horizon $\mathrm{T}=6$ is shown:

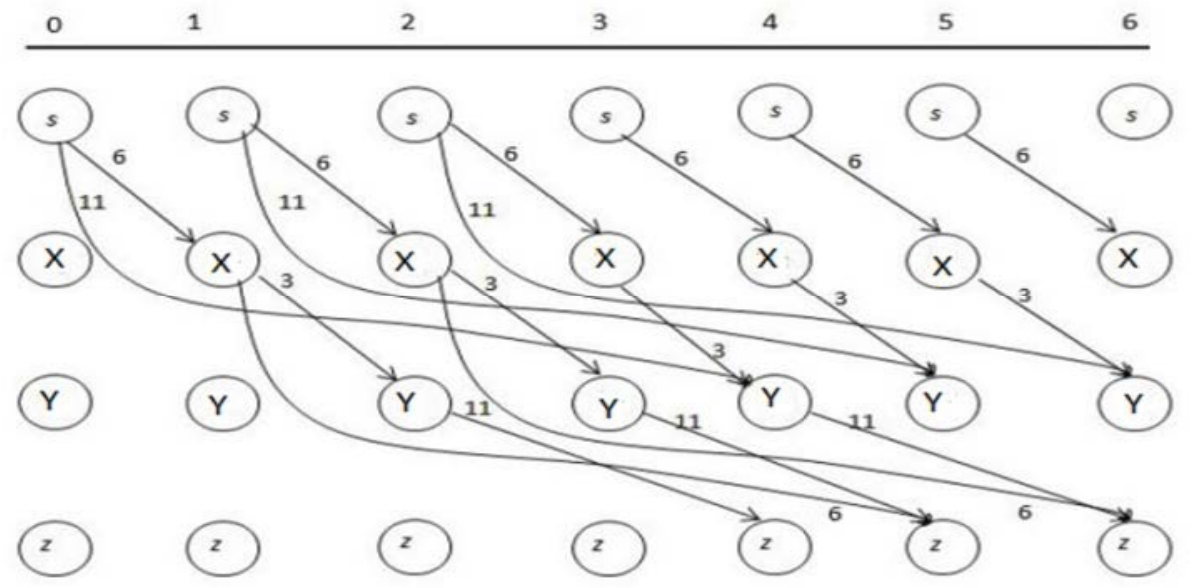


Fig 2 Time- expanded Graph of dynamic network figure 1 with time horizon $\mathrm{T}$

\section{Example}

The capacity of each arc is given in figure 1 and corresponds to the capacity of the original arc of the underlying dynamic network. The transit times are modeled implicitly. e.g. $\tau_{s X}=1$ is expressed in the time - expanded graph by the form of that arc always pointing from $\mathrm{s}(\mathrm{t})$ to $\mathrm{X}(\mathrm{t}+1)$. We have left out hold over arcs, which would just point from $i(t)$ to $i(t+1)$ for each node $i \in\{s, X, Y, z\}$ and each $t \in$ $\{0,1, \ldots, 6\}$.

A maximal flow on this time - expanded graph can be found in figure 1 by applying the usual static network flow theory such a maximal flow is presented in figure 2 . The total flow arriving at sink $\mathrm{z}$ within time horizon $\mathrm{T}=6$ is $\sum_{t=0}^{n 6} u(z(t))=23$.

Where 5 units of flow arrive at node $\mathrm{z}(5)$ and 15 units of flow arrive at node $\mathrm{z}(6)$. This corresponds to 5 units arriving at the sink at time $\mathrm{T}=5$ and 17 units arriving at the sink at time $\mathrm{T}=6$. Before time $\mathrm{T}=5$ no flow arrives at the sink $\mathrm{z}$.

The size of time-expanded graph is the major problem, which is dependent on the time horizon T. Thus any algorithm that works on the time - expanded graph can only be a pseudo - polynomial algorithm.

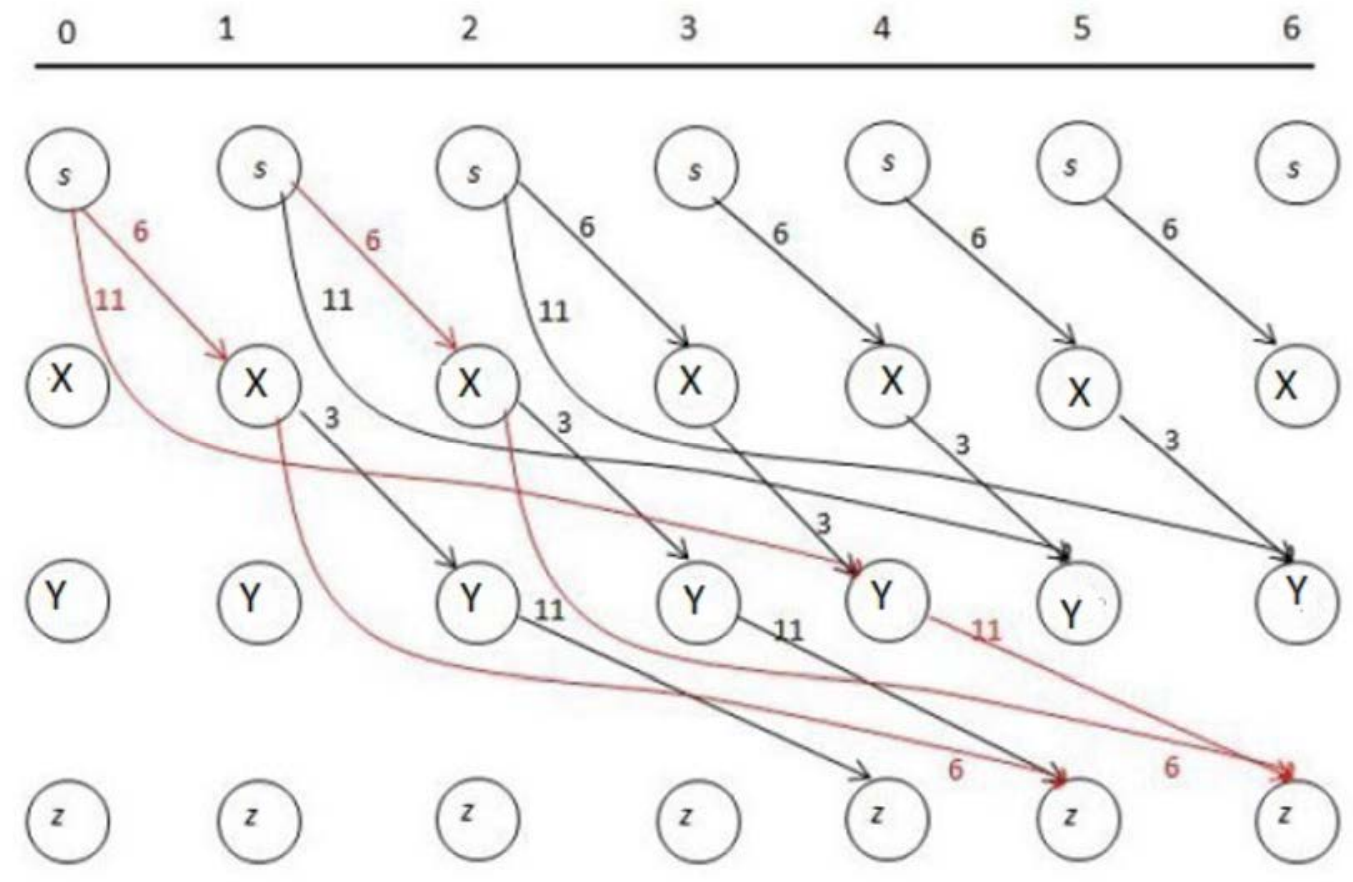

Fig 3 Earliest Arrival Flow of Fig 2.

\section{Earliest Arrival Flow Problem}

The objective is to send flow from source $\mathrm{s}$ to sink $\mathrm{z}$ that moves through the network over time such that for each point in time $t \in\{0, \ldots, T\}$, the maximum possible amount of flow reaches to sink $\mathrm{z}$. For each $\mathrm{t}$, if this flow is maximum flow for time horizon T, then it is called Earliest Arrival Flow.

That is the earliest arrival flows are those maximum dynamic $\mathrm{s}-\mathrm{z}$ flows that sends for each point in time $t \in\{0, \ldots, T\}$ the maximum amount of flow from $s$ to $z$. While the existence of a maximum flow for a fixed time horizon $\mathrm{T}$ is obvious, flows over time are not only optimal for $\mathrm{T}$ but also for 
each t. Gale showed the existence of earliest arrival flows for general networks with transit times on the arc and more generally, for networks with time -dependent (but not flow - dependent) transit times and capacity on the arcs. He used the fact that one can model flows over time with constant or time dependent transit times using static flows in the time - expanded network.

It is again obvious that for any time horizon $\mathrm{T}$ there exist a dynamic $\mathrm{s}-\mathrm{z}$ flow that sends the maximum amount of flow from $\mathrm{s}$ to $\mathrm{z}$. There is no simple time expanded model for dynamic flows with flow dependent transit times which reduce the time - dependent flow problem to a static network.

The formulation of earliest arrival flow problem was given by the D.Gale based on Maximum dynamic flow given by Ford and Fulkerson. The above formulation gives the earliest arrival flow if it gives maximum value of $v$ at each time $t$.

\section{The Complexity}

The flows in the given dynamic network are equivalent to the static flows in the time-expanded network. The algorithms based on the graphs are polynomial time in $\mathrm{T}$ and thus have pseudopolynomial running time. A polynomial time solution for the s-z maximum flow problem has been obtained by temporally repeated flows in the original graph. This makes use of the standard flow decomposition techniques where chains carry only positive flows.

The earliest arrival flow (EAF) is also known as universal maximum flow problem (UMEP) aims a solution such that the total number of evacuees reaching the destination is maximal for all points in time simultaneously. The s-z problem has been solved in pseudo-polynomial by successive shortest path algorithm for min-cost flow computation.

\section{Concluding Remarks}

In this survey we have studied the two dynamic flow problems, one of them is maximal dynamic flow problem another is earliest arrival dynamic flow problem. Both of them are used in evacuation problems. In this article we survey the earliest arrival flow problem, which is one of the important flow models in network optimization theory.

As we have seen, it is still an open problem if there exists a polynomial time algorithm for the earliest arrival flow problem or not, so this is the open question for further research.

\section{Acknowledgement}

The research of Shree Ram Khadka was supported by the European Commission in the framework of Erasmus Mundus and within the project cLINK and Kantipur Engineering College.

\section{References}

1. Arulselvan A., "Network model for disaster management", Ph D Thesis, University of Florida USA, (2009)

2. Baumann, $\mathrm{N}$ and Skutella,M , "Solving evacuation problems efficiently, Earliest arrival flows with multiple sources", Foundation of computer science FOCS \{2006, 47th\} Annual TEEE symposium, 399 - 410, (2006)

3. Bein,W.W. Brucker P. and Tamir A., "Minimum cost flow Algorithms for series parallel networks", Discrete applied Mathematics 10, 117-124, (1985) 
4. Brucker, P., "Network flows in trees and Knapsack problems with nested constraints" Proceeding on 8th Conference on graph theoretic concepts in computer science, Hanser 25$35,(1982)$

5. Dhamla, T.N., "A survey models algorithms for discrete evacuation planning network problems", Journal of Industrial and Management Optimization, 11(1),265-289, (2015)

6. Dhamla T.N. and Pyakurel U., "Earliest Arrival Contraflow Problem on series parallel graph”, International Journal of Operations Research, 10(1),1-13, (2013)

7. Ford,L.R. and Fulkersion D.R., "Constructing maximal dynamic flows from static network" Operations Research 6, 419-433, (1958)

8. Ford,L.R. and Fulkersion D.R., "Flows in networks", Princeton University Press,Princeton, New jersey, (1962)

9. Gale, D., "Transient flows in network”, Michigan Mathematical Journal, 659-663, (1959)

10. Hoppe, B.andTardos, E., "Polynomial time algorithm for some evacuation problems", Proceeding of 5th Annual ACM-SIAM symp. on Discrete Algorithm, (1994)

11. Hoppe, B., "Efficient dynamic network flow algorithms", Ph.D Thesis, Cornell University, (1995)

12. Rebennack, S., Arulselvan, A., Elefteriadou, L. and Pardolas, P. M., "Complexity analysis of maximum flow problem with arc reversal", "Journal of Combinatorial Optimization 29, 200-216, (2010)

13. Ruzika, S., Sperber, H. and Steiner, M., "Earliest arrival flows on series parallel graphs", Network 10, 169-173, (2011)

14. Steiner, M., "A survey on Earliest arrival flow and a study of the series parallel case Diploma thesis", Department of Mathematics, University of Kaiserslautern, (2009)

15. Wilkinson, W. L., "An algorithm for universal maximal dynamic flows in a network", Operations Research 19, 1602-1612, (1971)

16. L. Fleischer and M. Skutella, "The quickest multi commodity flow problem Integer programming and Combinatorial optimization", volume 2337 of Lecture Notes in computer science $36-53,(2000)$

17. Minieka E., "Maximal lexicographic and dynamic flows", Operations Research 21: 517$527,(1973)$ 